Int. J. Dev. Biol. 61: 267-276 (2017)

doi: $10.1387 / \mathrm{ijdb} .160442 \mathrm{yc}$

\title{
Roles of the cilium-associated gene CCDC11 in left-right patterning and in laterality disorders in humans
}

\author{
MICHAL GUR ${ }^{1}$, ENBAL BEN-TAL COHEN ${ }^{1}$, OLGA GENIN ${ }^{1}$, ABRAHAM FAINSOD ${ }^{2}$, ZEEV PERLES ${ }^{3}$ \\ and YUVAL CINNAMON*,1 \\ ${ }^{1}$ Agricultural Research Organization, The Volcani Center, Department of Poultry and Aquaculture Science, Rishon \\ LeTsiyon, ${ }^{2}$ Department of Developmental Biology and Cancer Research, Institute for Medical Research, Israel- \\ Canada, Faculty of Medicine, Hebrew University of Jerusalem, Jerusalem and ${ }^{3}$ Pediatric Cardiology Department, \\ Division of Pediatrics, Hadassah - Hebrew University Medical Center, Jerusalem, Israel
}

\begin{abstract}
Axial determination occurs during early stages of embryogenesis. Flaws in laterality patterning result in abnormal positioning of visceral organs, as manifested in heterotaxy syndrome, or complete left-right inversion as in situs inversus totalis. These malformations are often associated with ciliopathies, as seen in primary ciliary dyskinesia. We have recently described a novel mutation in the Coiled-Coil Domain-Containing 11 (CCDC11) gene associated with laterality disorders in a consanguineous family of Arab-Muslim origin with two affected siblings presenting with diverse phenotypes, one with heterotaxy syndrome and the other with non-primary ciliary dyskinesia situs inversus totalis. This study further characterizes the roles of $C C D C 11$ and the implications of the identified mutation on left-right axial patterning in patient-derived cells and in the frog embryo as a model organism. We analyzed patient-derived cells and manipulated Ccdc11 levels in Xenopus laevis frog embryos. Cilia length in patient cells was longer than in controls, and CCDC11 was localized to the centriole and the actin cytoskeleton. Mutated truncated protein accumulated and was also localized to the centriole and actin cytoskeleton. In frog embryos, Ccdc11 was regulated downstream of FoxJ1, and overexpression of the full-length or truncated protein, or downregulation of the gene resulted in severe disruption of embryonic left-right axial patterning. Taken together, our initial description of the deleterious mutation in $C C D C 11$ in patients, the current results and more recent supportive studies highlight the important role of CCDC11 in axial patterning.
\end{abstract}

KEY WORDS: CCDC11, cilia, laterality disorder, human mutation, Xenopus laevis embryo

\section{Introduction}

The orthogonal axes of the body are determined during early stages of embryogenesis. Vertebrate organisms appear bilaterally symmetrical from the outside, but all exhibit visceral left-right $(\mathrm{L}-\mathrm{R})$ asymmetry known as situs solitus (Levin, 2005). While proper patterning of the body axes is obligatory for survival, flaws in L-R asymmetry can be viable. Failure to generate normal asymmetry may result in several morphological anomalies, among them heterotaxy syndrome (HS) and situs inversus totalis (SIT) (Perloff, 2011). HS, also called situs ambiguous, is abnormal $L-R$ axis arrangement of the abdominal and/or thoracic viscera, involving organs which normally develop on one side of the body. Most HS patients present with complex congenital heart disease, which is often fatal (Bartram et al., 2005; Brueckner, 2007). SIT is a mirror- image asymmetry of the internal abdominal and thoracic viscera and is frequently associated with Kartagener syndrome (Kartagener and Stucki, 1962) or primary ciliary dyskinesia (PCD) (Leigh et al., 2009). In rare situations, SIT may occur as an isolated anomaly with otherwise normal anatomy and organ function. The first link between situs anomalies and ciliary dysfunction was identified by Afzelius (1976), describing a high proportion of patients with sperm immotility and chronic sinusitis who also had SIT. It was later found

\footnotetext{
Abbreviations used in this paper: CCDC, coiled-coil domain-containing; GFP, green fluorescent protein; GRP, gastrocoel roof plate; HS, heterotaxy syndrome; IHC, immunohistochemistry; ISIT, isolated situs inversus totalis; L-R, left-right; $\mathrm{MO}$, morpholino oligonucleotide; PCD, primary ciliary dyskinesia; SIT, situs inversus totalis; qPCR, quantitative reverse-transcription PCR; St., Xenopus laevis embryonic stage; WT, wild type.
}

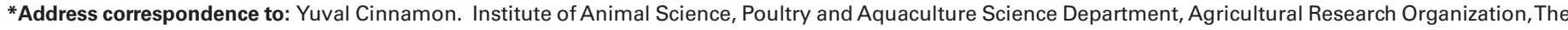
Volcani Center, HaMaccabim Road, P.O. Box 15159, Rishon LeTsiyon 7528809, Israel. E-mail: yuval.cinnamon@mail.huji.ac.il Web: http://www.agri.gov.il/people/1122.aspx - (iD) http://orcid.org/0000-0002-6234-8219
}

Accepted: 12 December, 2016.

ISSN: Online 1696-3547, Print 0214-6282 
that the nodal cilia indeed play a pivotal role in the establishment of L-R asymmetry in mice (Nonaka et al., 1998), fish (Essner et al., 2005) and frogs (Blum et al., 2007) as well.

Mutations in several cilium-associated genes, mostly encoding dynein arm components, such as DNAI1 and DNAH5, have been found to be involved incausing PCD (Kennedy etal., 2007). However, the list of mutated genes that cause $\mathrm{L}-\mathrm{R}$ asymmetry defects not associated with PCD is much shorter. Using a homozygosity-mapping approach, we previously identified and described a homozygous deleterious mutation in the gene Coiled-Coil Domain-Containing 11 (CCDC11, MIM\# 614759, HGNC gene symbol: CFAP53) in a consanguineous family of Arab-Muslim origin (Perles et al., 2012). Two homozygous sibling patients were identified, remarkably presenting with dramatic clinical variance. The first brother was admitted to our tertiary care facility due to a severe and complex congenital cardiovascular defect and abdominal situs anomalies, and was therefore diagnosed with HS. The patient underwent bidirectional superior cavopulmonary connection (Glenn procedure), but there were postoperative complications, leading to his death. The second homozygous brother, with SIT, was apparently healthy and consistent with the lack of any clinical symptoms, his nasal-brushing sample revealed normally beating ciliated cells as well as normal ciliary ultrastructure, as determined by electron microscopy. Furthermore, sperm examination revealed normal ejaculation volume, count and motility, indicating a normal content and function of motile cilia. Hence, having no signs of PCD, this sibling was diagnosed with isolated SIT (ISIT).

$C C D C 11$ consists of eight exons that encode a 514-amino acid protein. The mutation that we identified in CCDC11 resulted in disruption of a splice site which led to skipping of the sixth exon. Consequently, exon 7 was introduced out of frame, directly following exon 5. This resulted in an introduction of 17 non-synonymous amino acids followed by a premature stop codon. Furthermore, as predicted from the sequence of the mutated cDNA transcript, we showed that a truncated protein lacking the C-terminal part, downstream of the premature stop codon, is translated. This truncated form of CCDC11 was found accumulated in the ISIT patient's skin fibroblast and nasal epithelium-derived cells (Perles et al., 2012).

Following the initial description of the role of CCDC11 in L-R patterning, more patients harboring mutations in $C C D C 11$ were described in the literature (Narasimhan et al., 2015; Noël et al., 2016), supporting CCDC11's involvement in axial patterning. To better understand this role, here we describe a study of skin fibroblast cells from the ISIT patient as well as a functional study in frog embryos.

Rapid embryonic development, hundreds of eggs per lay and the ability to manipulate gene expression make the Xenopus laevis frog embryo an ideal organism to study embryonic development and axial patterning. Establishment of the $\mathrm{L}-\mathrm{R}$ axis and symmetry breakage have been shown to be evolutionarily conserved among vertebrates, and the involvement of cilia in this process has been demonstrated in mice, rabbit, zebrafish and frog embryos (Blum et al., 2014; Raya and Izpisúa Belmonte, 2006; Wessely and Obara, 2008). In that sense, the $X$. laevis has been found to be useful for modeling pathogenic mutations involved in L-R patterning (Endicott et al., 2015).

In frog embryos, the initiation of symmetry breakage occurs in the gastrocoel roof plate (GRP) which has been identified as the L-R organizer, with characteristics similar to those of the mouse node and Kupffer's vesicle in zebrafish (Essner et al., 2005; Nonaka et al., 1998; Schweickert et al., 2007). These structures contain monociliated epithelial cells, which create a leftward flow by synchronized beating, eventually affecting the lateral expression of Nodal (Blum et al., 2007; Schweickert et al., 2010; Vick et al., 2009). Derived from the superficial mesoderm (Shook et al., 2004), the GRP is relatively large in size and the cells' fate is well characterized. Hence, this structure is the subject of gain- and loss-of-function experiments by targeted microinjection of mRNAs, antisense morpholino oligonucleotides (MOs), plasmids and other small molecules (Blum et al., 2009). GRP explants can be analyzed by live imaging systems for cilium-mediated fluid flow or by histological protocols for cilium structure and function (Schweickert et al., 2007).

The Forkhead transcription factor Foxj1 is a master regulator of ciliogenesis (Yu et al., 2008) and is therefore essential for L-R patterning (Stubbs et al., 2008). In the frog, xFoxj1 is expressed in the superficial mesoderm during gastrulation, in the GRP and in the ciliated epithelial cells (Pohl and Knöchel, 2004; Stubbs et al., 2008). Downregulation of xFoxj1 results in loss or severe shortening of the cilia, whereas overexpression induces ectopic formation of cilia. The extracellular leftward flow formed by the GRP cilia breaks the bilateral symmetry by removing repression of the Nodal signaling cascade on the left side (Hirokawa et al., 2006; Nonaka et al., 1998; Schweickert et al., 2007). This process is mediated by the Nodal inhibitor xCoco (DAND5). xCoco is initially expressed uniformly on both sides of the GRP; however as a result of the leftward flow, its expression on the left side declines, thereby removing the inhibition and allowing for activation of Nodal signaling on this side (Schweickert et al., 2010). Thus, besides being crucial for proper laterality formation, $x$ Cocoexpression pattern along the GRP, which can be easily detected by RNA in-situ hybridization staining, serves as the earliest known marker of symmetry breakage in the frog. As a later definitive marker for laterality, the homeobox transcription factor $x$ Pitx2 can be used. $x$ Pitx 2 is regulated downstream of the Nodal signaling pathway and is required for $L-R$ patterning of internal organs in vertebrate embryos (Campione et al., 1999; Ryan et al., 1998). $x P$ Pit $x 2$ is normally expressed unilaterally on the left lateral plate mesoderm, thus serving as a reliable late marker for laterality and laterality disorders, once L-R patterning has been established.

Here, we present a study of ISIT patient-derived cells showing that the cilia in these cells are longer than in controls and that CCDC11 is localized to the centriole as well as to the actin cytoskeleton. Using western blot analysis, we previously showed that the truncated form of CCDC11 accumulates in the patient's cells (Perles et al., 2012). Here we show that this accumulating protein localizes to the actin cytoskeleton. Furthermore, we show that in the frog embryo, $x C c d c 11$ is regulated downstream of $x F o x J 1$, and that overexpression of the full-length or truncated protein, or downregulation of the gene results in severe disruption of embryonic $\mathrm{L}-\mathrm{R}$ axial patterning.

\section{Results}

CCDC11 localizes to the centriole in cultured skin fibroblast cells, and the mutant truncated protein colocalizes to the actin cytoskeleton in patient-derived cells

CCDC11 was initially identified in a consanguineous family with two siblings homozygous for a splice-site mutation presenting defects in L-R patterning, suggesting a role for CCDC11 in 
ciliogenesis or ciliary function. Using electron microscopy analysis of a nasal brush biopsy taken from the ISIT patient, we previously confirmed an intact respiratory ciliary axonemal ultrastructure (Perles et al., 2012). To study whether the mutation in CCDC11 is involved in other aspects of ciliogenesis, we immunostained control and patient monociliated skin fibroblasts with anti-ARL13B, a cilium-specific antibody (Fig. 1 A,B) and determined the proportion of ciliated cells in culture as well as cilium length. Quantification of the number of ciliated cells showed that in fibroblasts from the ISIT sibling there was a significant (23\%) reduction, from $68 \%$ to $52 \%$, in the number of ciliated cells (a total of 85 and 74 cells were counted, respectively), while their average length increased by $0.3 \mu \mathrm{m}$ compared to controls $(P<0.01$, Fig. $1 \mathrm{C})$. A similar scale of change in cilia length and frequency has been shown to be clinically relevant in ciliopathies (Walczak-Sztulpa et al., 2010). Our ISIT patient, however, showed no clinical symptoms. Furthermore, immunohistochemical (IHC) staining of skin fibroblasts with antiCCDC11 antibody confirmed our previous finding of a relatively low level of CCDC11 expression in control cells, whereas the mutated transcript results in high accumulation of truncated CCDC11 protein in patient cells (Fig. 1 D-G). Interestingly, in control cells, CCDC11 was localized to the centriole and to a much lesser extent to the actin cytoskeleton (Fig. 1 D,F). However, in the ISIT patient's cells, the truncated protein colocalized to the actin cytoskeleton as well as to the centriole (Fig. $1 \mathrm{E}, \mathrm{G}$ ). Taken together, our results show that in human skin fibroblast cells, CCDC11 is associated with the centriole and the actin cytoskeleton and the truncated protein accumulates.

\section{Expression pattern of Xenopus leavis CCDC11 (xCCDC11) during left-right determination stages in ciliated cells}

Using the frog model, we studied the role of the $X$. laevis CCDC11 ortholog $(x C c d c 11)$ in $\mathrm{L}-\mathrm{R}$ patterning. Frog embryos have been widely used as a model system for axial patterning research as gene-expression analysis and gene manipulation are possible using a range of available tools (Blum et al., 2009). To evaluate the temporal expression of $x C c d c 11$ during early stages of embryogenesis, we performed a quantitative reverse-transcription PCR (qPCR) study on successive embryonic stages (St.) from the mid-blastula transition (St. 8) (Nieuwkoop and Faber, 1975) to organogenesis (St. 24) (Fig. 2A). We showed that $x$ Ccdc11 expression begins at the gastrula stage (St. 10.5), increases during neurulation and continues to accumulate through organogenesis (Fig. 2A). In frogs, breakage of bilateral symmetry depends on synchronized beating of motile cilia at the GRP at St. 17 (Schweickert et al., 2007; Vick et al., 2009). The GRP is derived from

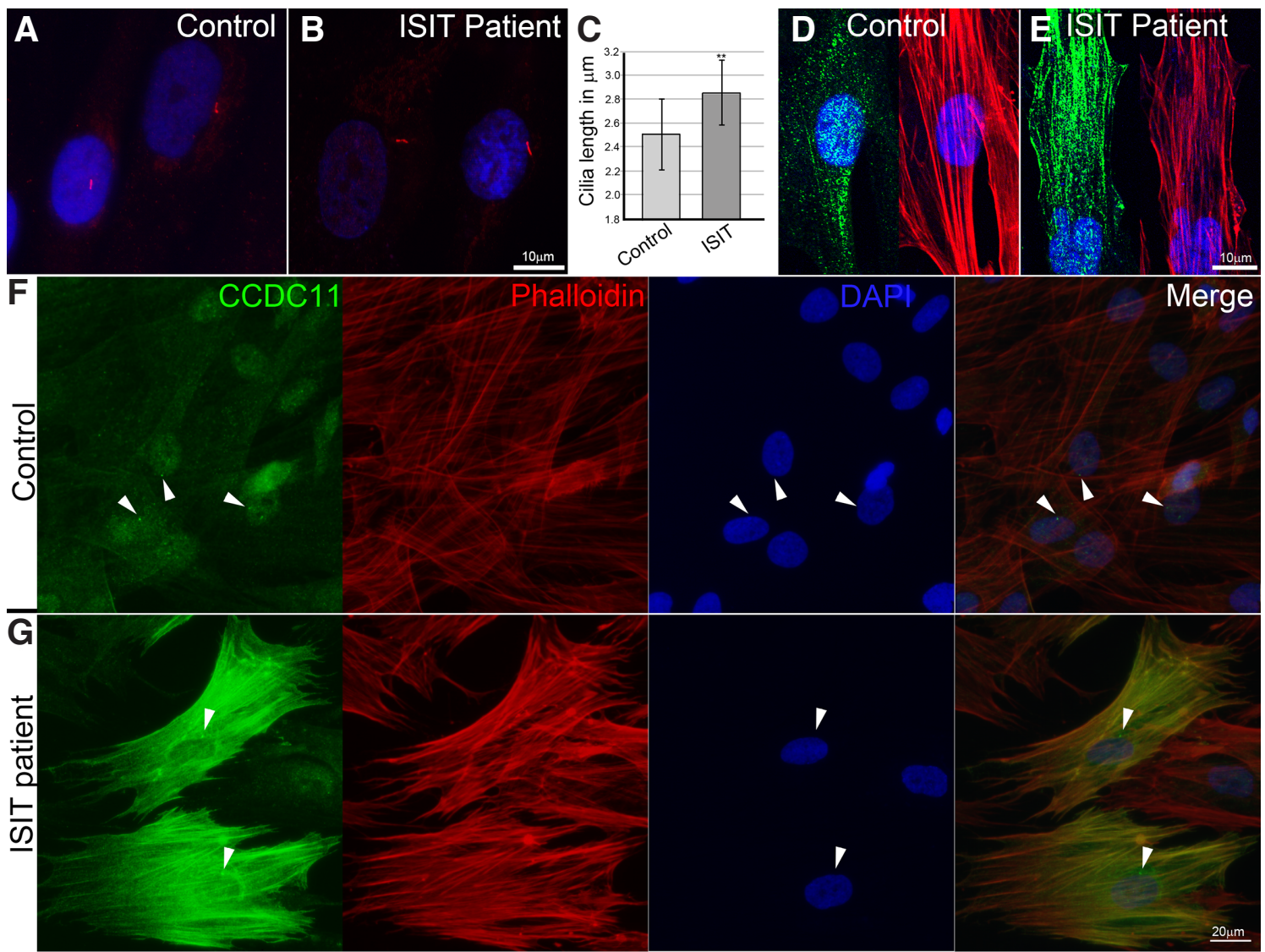

Fig. 1. Ciliogenesis analysis of patient-derived monociliated skin fibroblast cells and subcellular localization of CCDC11. (A,B) Immunostaining with anti-ARL13B antibody (red) and DAPI nuclear staining (blue). Representative images of cilia showing longer cilia in patient's fibroblasts. (C) Cilium length was measured and compared between groups. Average cilium length in patient's cells was significantly longer (by $0.3 \mu \mathrm{m} ; \mathrm{P}<0.01)$. (D,F) Control and (E,G) patient cells were stained with rhodamine phalloidin (red), anti-CCDC11 antibody (green) and DAPI (blue). While in control cells, only a minute amount of CCDC11 was localized to the actin cytoskeleton (D), in the patient cells, where truncated protein accumulates, the protein was widely localized to the stress fibers $(\mathbf{E}, \mathbf{G})$. Both WT and mutated proteins were also localized to the centriole (arrowheads in F and G). 
A
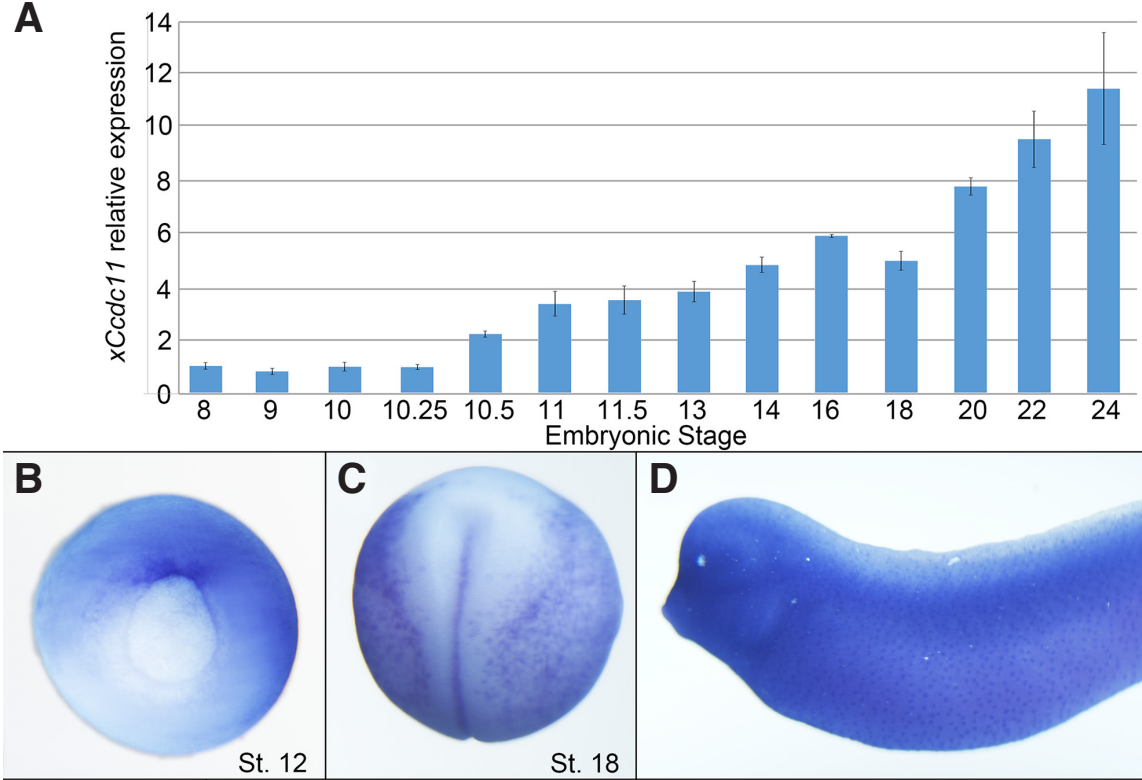

D
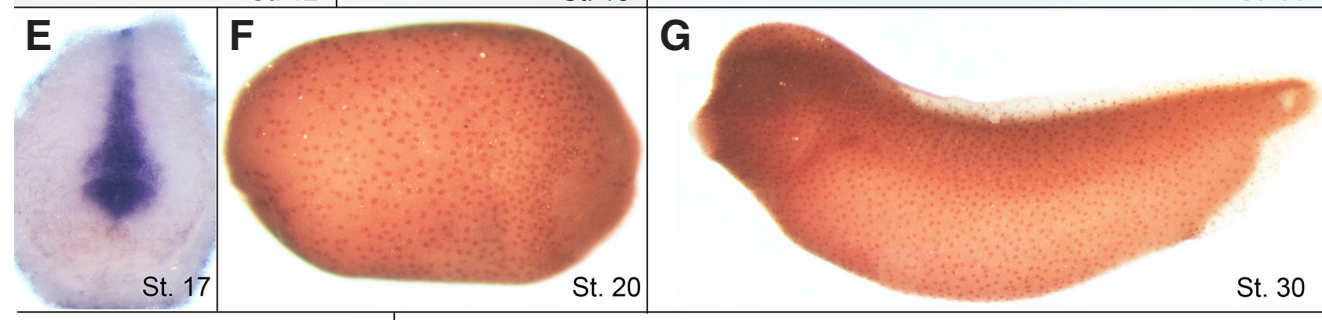

$\mathbf{H}$

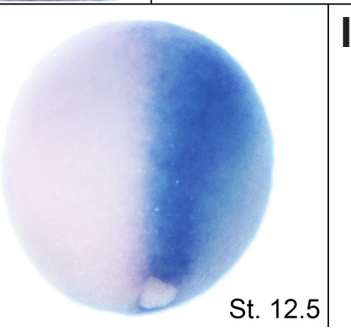

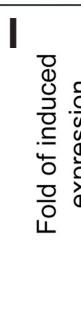

G

St. 20
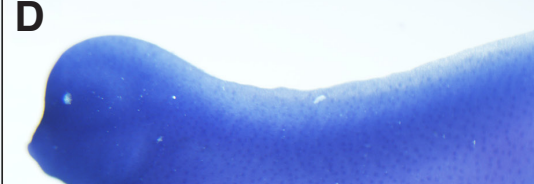

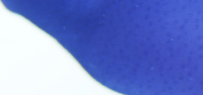
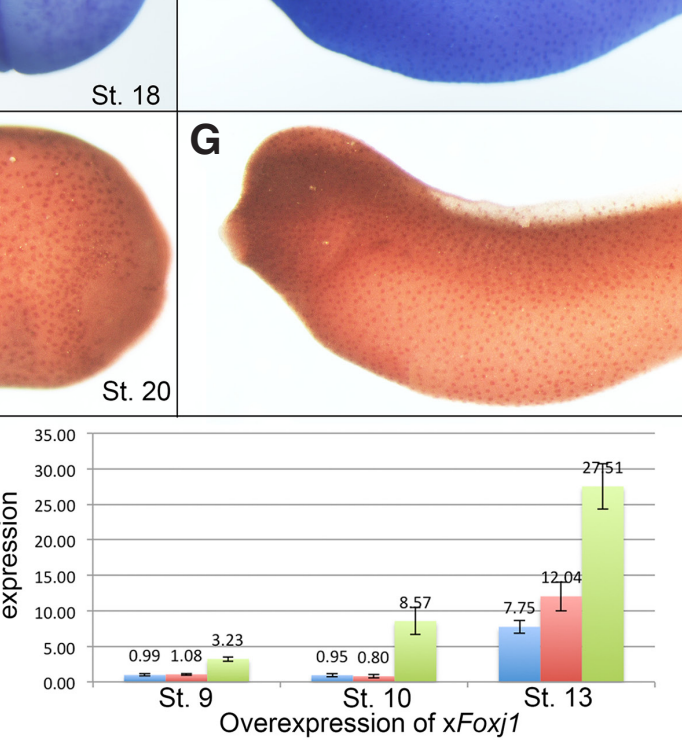

Fig. 2. Spatiotemporal expression of Ccdc11 in Xenopus laevis and its regulation by FoxJ1. To determine the temporal expression pattern of $\mathrm{xCcdc} 11$, embryos were collected at different developmental stages from blastula to early tailbud and level of expression was determined by qPCR (A). Temporal expression shows that $\mathrm{XC} \mathrm{Cdc} 11$ starts to be expressedat gastrulation (St. 10.5), increases during neurulation and continues to accumulate through development. (B) Spatial expression of $\mathrm{xCcdc} 11$ by wholemount in-situ hybridization shows that at late gastrulation, $\mathrm{xCcdc11}$ is expressed in the dorsal lip of the blastopore (St. 12). (C) At neurulation (St. 18) and (D) at the tailbud stage (St. 28), embryo staining shows a scattered pattern of ectodermal layer, stereotypic to ciliated cells. (E) Section of neurula embryo shows expression at the GRP (St. 17). IHC staining with anti-CCDC11 antibody shows similar expression patterns in the ectodermal layer in (F) late neurula (St. 20) and (G) tailbud (St. 30). Breakage of bilateral symmetry in frogs depends on the development of ciliated epithelium at the GRP, emerging from the dorsal lip of the blastopore. The qPCR and in-situ hybridization assays gave a spatiotemporal pattern of $\mathrm{x} C \mathrm{Cdc} 11$ expression that suggests a role in ciliogenesis and in axial patterning. $(\mathbf{H}, \mathbf{I}) \times \mathrm{Ccdc} 11$ expression is regulated by $\mathrm{xFoxj} 1$. The Forkhead transcription factor Foxj1 is a master regulator gene of motile cilia. To test its regulatory effect on $\mathrm{xCcdc} 11$ expression, embryos were injected at 2- to 4-cell stage with xFoxj1 RNA.

For in-situ hybridization analysis, embryos were injected unilaterally with xFoxj1 RNA together with FITC, as lineage tracer, and collected at St. 12.5. In-situ hybridization staining shows that on the injected side (right, marked by blue staining), xCcdc11 levels are dramatically upregulated compare to the uninjected side, which serves as an internal control (H). For quantitative analysis, embryos were injected radially and total RNA was extracted at St. 9, 10 and 13. qPCR shows that similar to other genes expressed in the GRP and critical for cilia motility (xTekt2 and xDnah9), xCcdc11 expression increases with xFoxj1 overexpression, but is not sufficient to induce ectopic expression prior to gastrulation (I). Both qPCR and in-situ hybridization analyses indicate that the master regulator of motile cilia, FOXJ1, positively regulates Ccdc11 expression, suggesting a role for CCDC11 in ciliogenesis.

the superficial mesoderm and motile cilia developed through early neurula. Thus, $x C c d c 11$ expression during gastrula and neurula correlates with the initiation of axial patterning (Fig. 2A). Next, we cloned the full coding sequence of $x C c d c 11$ and prepared probes for RNA in-situ hybridization staining on embryos from gastrula, neurula and tailbud stages (Fig. $2 \mathrm{~B}-\mathrm{D}$, respectively). The in-situ staining confirmed the qPCR results and demonstrated that during gastrulation, $x C c d c 11$ is expressed at the dorsal lip of the blastopore, the superficial mesoderm that will give rise to the GRP (St. 12, Fig. 2B). Dissection of embryos during neurulation confirmed $x C c d c 11$ expression at the GRP itself (St. 18, Fig. $2 \mathrm{C}$ - intact neurula and 2E - GRP). Tailbud embryos (St. 30) showed a scattered pattern of expression in epidermal cells which is stereotypical of motile ciliated cells (Vick et al., 2009) (Fig. 2D). Correspondingly, IHC staining with anti-CCDC11 antibody revealed a similar ectodermal expression pattern (Fig. 2 F,G). Taken together, the spatiotemporal expression pattern was concordant with a role in $L-R$ patterning and supports our notion that CCDC11 has an evolutionarily conserved role in this process.

\section{$x$ Foxj1 regulates $x C c d c 11$ expression from the onset of gastrulation}

The forkhead transcription factor Foxj 1 is a known master regulator of motile cilia and has been shown to drive ciliogenesis in multiciliated cells (Stubbs et al., 2008). Major ciliary genes such as Tekt2 and Dnah9 are known downstream targets of Foxj1 (Yu et al., 2008). The expression pattern of $x C c d c 11$ in ciliated cells resembles that of $x$ Foxj1 (Pohl and Knöchel, 2004), and recessive mutations in FOXJ1 have been shown to be involved in laterality defects in patients. Thus, we studied whether $x$ Foxj1 regulates 
$x C c d c 11$ expression by overexpressing $x$ Foxj1 in embryos. Fourcell-stage embryos were injected on one side with xFoxj1 mRNA and were allowed to develop until St. 12.5, when they were stained for $\mathrm{x} C c d c 11$ expression by in-situ hybridization. These one-sided injections led to ectopic and strong overexpression of $\mathrm{x} C \mathrm{cdc} 11$ on the injected side (Fig. $2 \mathrm{H}$ ). To quantify this upregulation, radial $x$ Foxj 1 mRNAinjections were performed; the embryos were allowed to develop and collected during late blastula (St. 9), at the onset of gastrulation (St. 10) and in early neurula (St. 13). qPCR analysis of these embryos revealed no change in expression levels at St. 9 vs. St. 10; by St. 13, qPCR analysis revealed 7.75-fold upregulation of $x C c d c 11$ expression level compared to controls. Similarly, as a positive control, the expression of both $x$ Tekt2 and xDnah9 was shown to be upregulated by xFoxj1 overexpression (Fig. 2l).
Thus, we confirmed that like $x$ Tekt2 and $x$ Dnah9, which are also expressed in the GRP and are critical for cilium motility (Stubbs et al., 2008), xCcdc11 expression is positively regulated by Foxj1 from the gastrulation stage. However, prior to gastrula stage, before the endogenous onset of $x C c d c 11$ expression, $x$ Foxj1 overexpression by itself is not sufficient for $x C c d c 11$ ectopic or untimely activation, suggesting more complex regulation of the latter.

\section{xCcdc11 downregulation impairs early and late left-right patterning}

To study the function of $x$ Ccdc11 in axial patterning, we generated an antisense $\mathrm{MO}\left(\mathrm{xCcdc}_{11-\mathrm{MO})}\right.$ to target the initiator methionine in the coding sequence, thereby facilitating $x$ Ccdc11 knockdown. To test its efficiency at blocking translation of $x$ Ccdc11 mRNA,

Fig. 3. Misexpression of $x$ Ccdc11 impairs L-R patterning. To study the role of CCDC11 in frogs, we knocked down its expression using xCcdc11-MO. Embryos were injected at the 2- to 4-cell stage and analyzed for $x$ Coco expression by whole-mount in-situ hybridization on St. 20 GRPs. Embryos injected with control-MO or uninjected embryos served as controls. In normal development, xCoco expression on the left is inhibited (blue arrowhead in $\mathbf{A})$, while it continues to be expressed on the right (A). When leftward flow is defective, $\mathrm{xCoco}$ expression may be either uniform, reflecting no inhibition at all (B), or reduced on the right side (blue arrowhead in C). Analysis of embryos injected with xCcdc11MO revealed disruption in the proper inhibition of $\mathrm{xCoco}$ expression. While only $25 \%$ of the embryos injected with control-MO exhibited deviation from normal expression, 49\% of $x \mathrm{Ccdc11-MO-injected}$ embryos showed abnor-
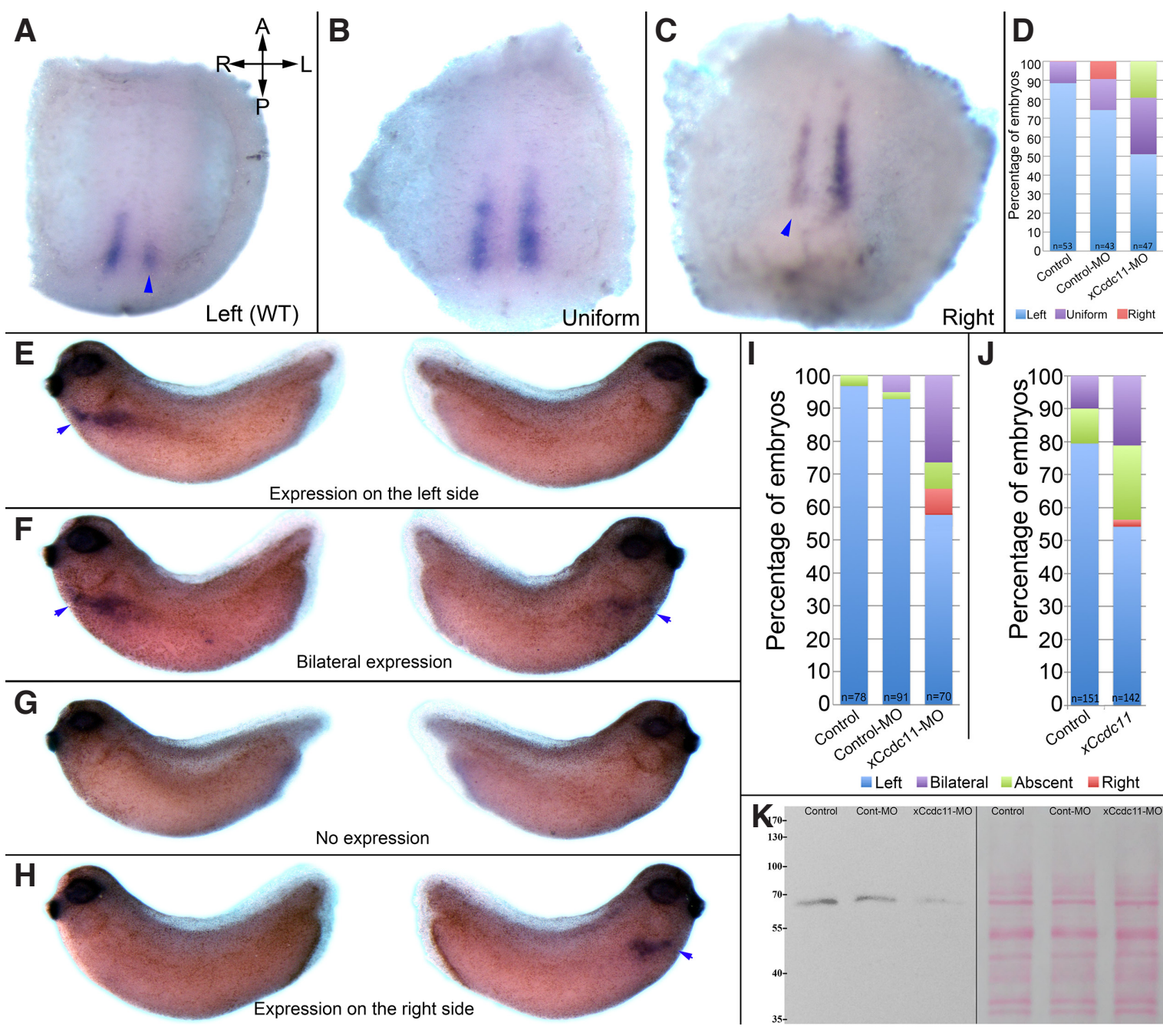

No expression
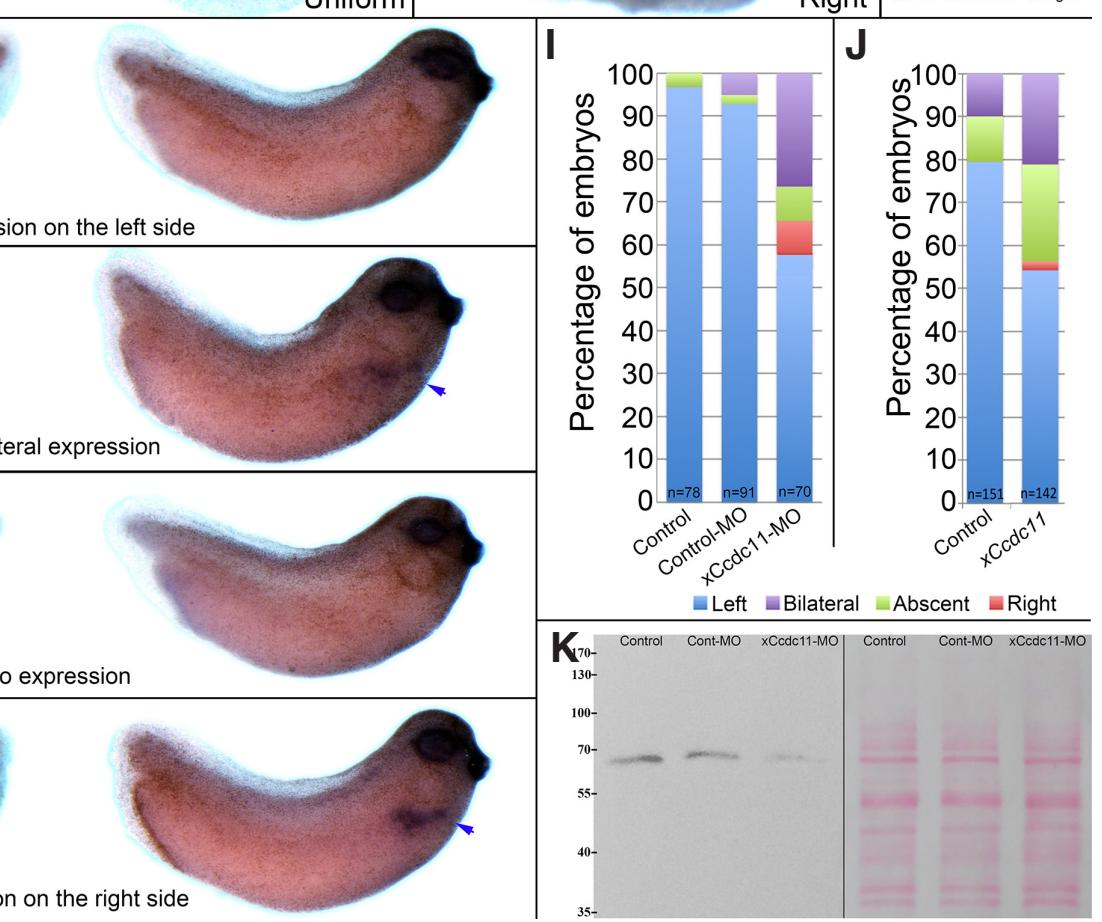

mal expression $(\mathrm{P}<0.05)$. To verify that the laterality disorders induced by $x$ Ccdc11 knockdown persist with time, we manipulated $x C c d c 11$ levels and evaluated $L-R$ patterning by in-situ hybridization staining for xPitx2 during the tailbud stages (blue arrowheads in $\mathbf{E}-\mathbf{H})$. xPitx2 is normally expressed unilaterally on the left, thus it serves as a reliable marker for laterality (E). Laterality defects may result in either bilateral (F), completely absent (G) or right expression (H) of xPitx2. Analysis of xCcdc11 downregulation by xCcdc11-MO injections resulted in laterality defects, as exhibited by abnormal expression of $x$ Pitx2 (I, P < 0.001). The ambiguous phenotype obtained in xCcdc11 knockdown mimics the diverse phenotype between the two siblings carrying the mutation, and is often observed when GRP flow is disrupted. Overexpression of $x \mathrm{Ccdc} 11$ also resulted in abnormal expression of $\mathrm{xPit} \times 2$ $(\mathbf{J}, \mathrm{P}<0.01)$, indicating the need for tight regulation of CCDC11 levels for proper $L-R$ patterning. The efficiency $x C c d c 11-M O$ in blocking translation of $\mathrm{xCcdc11}$ mRNA was assessed by western blot analysis of total proteins extracted from embryos injected with xCcdc11-MO, uninjected embryos, and embryos injected with control-MO. Analysis with anti-CCDC11 antibody revealed that XCcdc11-MO inhibits xCcdc 11 expression by $84 \%$ compared to control-MO-injected embryos. Ponceau S total protein staining of the same membrane was used to validate equal loading in all lanes (K). 


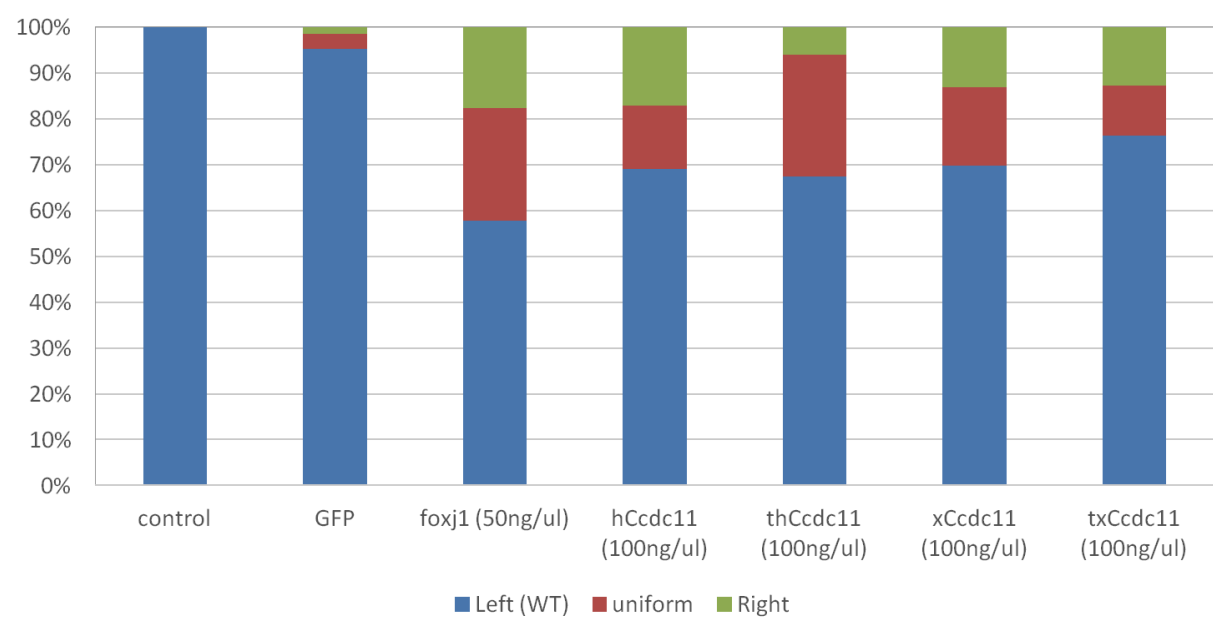

Fig. 4. Ccdc11 overexpression impairs L-R patterning. The accumulation of truncated CCDC11 in skin fibroblastand nasal epithelial cells from the ISIT sibling implies tight regulation and suggests that high levels of CCDC11 might interfere with normal ciliary function. To test this hypothesis, embryos were injected with mRNA encoding either the WT or the truncated form of Ccdc11 of both human and frog orthologs (hCcdc11, thCcdc11, xCcdc11 and $t \times C c d c 11$ respectively). Embryos injected with mRNA for GFP and xFoxj1 served as controls. To verify dorsal injection, mRNA encoding GFP was added and embryos were screened at early neurula stages. Embryos were collected at St. 20 and laterality was assessed byin-situ hybridization for $x$ Coco expression (as shown in Fig. 3). All four

forms of Ccdc11 overexpression resulted in abnormal xCoco expression, as with xFoxj1 overexpression. The disruption of proper axial patterning following CCDC11 misexpression indicates an essential role for CCDC11 in L-R patterning, and the prominence of controlled levels of expression.

embryos were radially injected with xCcdc11-MO. These embryos were compared with two control groups: uninjected and controlMO-injected embryos. The embryos were allowed to develop to the gastrula stage, and total protein was then extracted and separated by SDS-PAGE (equal loading per lane confirmed by Ponceau S total protein staining). Western blot analysis with anti-CCDC11 antibody revealed that $\mathrm{xCcdc} 11-\mathrm{MO}$ inhibits $\mathrm{xCcdc} 11$ expression by $84 \%$ compared to control-MO-injected embryos (Fig. $3 \mathrm{~K}$ ).

To study the effect of $x C c d c 11$ knockdown on L-R patterning during initiation of bilateral symmetry breakage, we analyzed the expression pattern of the transcription factor $x$ Coco which is the first known molecular marker for L-R asymmetry expressed in the GRP. The expression pattern of $x \mathrm{Coco}$ is dynamic during the early stages of development, and it is highly expressed in the animal cap at as early as the 4-cell stage. This expression declines rapidly between St. 10 and St. 11. Prior to leftward flow, $x$ Coco is uniformly expressed on both sides of the GRP. However, following the normal leftward flow created by ciliated cells in the GRP, $x$ Cocoexpression on the left side is reduced (Fig. 3A, arrowhead pointing to the left side, where $x$ Coco expression is reduced), while its expression on the right side continues. When motile cilia in the GRP are malformed or dysfunctional, the leftward flow is interrupted. This may result in bilaterally uniform expression reflecting no inhibition (Fig. 3B) or reverse expression, in which $x$ Coco inhibition occurs on the right side of the embryo while high expression is maintained on the left side (Fig. 3C, arrowhead pointing to the right side, where Coco expression is reduced).

The effect of $x$ Ccdc11 knockdown on $x$ Coco expression was analyzed by injecting $8 \mathrm{pmol} / \mathrm{embryo}$ of either control-MO or xCcdc11-MO. In addition, uninjected embryos were used as controls. The embryos were radially injected at the 2- to 4-cell stage, and were allowed to develop until St. 20. Dissected GRPs were analyzed for $x C o c o$ expression by whole-mount in-situ hybridization (Fig. $3 \mathrm{~A}-\mathrm{C}$, anterior-posterior and $\mathrm{L}-\mathrm{R}$ orientation of the embryos is indicated by arrows in A). Analysis of embryos injected with $\times C c d c 11-\mathrm{MO}$ revealed significant changes from the expected inhibition of $x$ Coco expression in the wild type (WT). While $25 \%$ of the embryos injected with control-MO exhibited deviations from

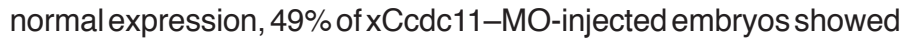
abnormal expression $(P<0.05$, Fig. 3D). These results indicate that $\mathrm{xCcdc11}$ plays a role in bilateral symmetry breakage and in defects in leftward flow in the GRP.

The asymmetric expression of $x$ Coco marks the onset of a cascade of processes that will eventually give rise to breakage of $L-R$ symmetry. Thus, to evaluate the effect of $x C c d c 11$ knockdown on late stages of $L-R$ patterning, we injected embryos with $x C c d c 11-M O$ and analyzed them for $x$ Pitx 2 expression during organogenesis. The homeobox transcription factor xPitx2 determines L-R asymmetry of internal organs in vertebrate embryos and is regulated downstream of the Nodal signaling pathway. $x$ Pitx 2 is expressed unilaterally on the left lateral plate mesoderm and therefore serves as a reliable marker for sidedness during organogenesis (Campione et al., 1999; Ryan et al., 1998). Embryos were radially injected at

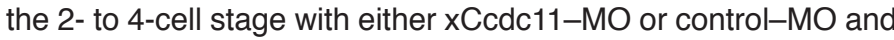
were allowed to develop until the tailbud stage. Uninjected embryos served as controls. Tailbud-stage embryos were then analyzed for $x$ Pitx2 expression by whole-mount RNA in-situ hybridization and four distinct expression patterns were scored: normal left-sided expression (Fig. 3E), bilateral expression (Fig. 3F), absence of expression on both sides (Fig. $3 \mathrm{G}$ ) and inversed, right lateral plate mesoderm expression (Fig. $3 \mathrm{H}$ ). Quantification of these results showed that while more than $90 \%$ of control uninjected embryos or embryos injected with control-MO presented normal expression of $x$ Pitx2, i.e., only on the left lateral plate mesoderm, only $59 \%$ of

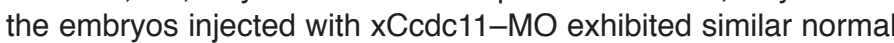
expression. The remaining $41 \%$ presented abnormal laterality patterns: $27 \%$ with bilateral expression of $x$ Pitx2, $7 \%$ with inversed expression on the right side and $7 \%$ with no $x$ Pit $x 2$ expression at all (Fig. 3I). These findings demonstrate laterality defects in advanced stages of organogenesis and highlight the role of $\mathrm{xCcdc11}$ in axial patterning.

\section{CCDC11 overexpression impairs left-right patterning}

The accumulation of truncated CCDC11 protein in skin fibroblasts and nasal epithelial cells from the ISIT sibling, as illustrated by western blot analysis (Perles et al., 2012) and IHC staining (Fig. $1 \mathrm{E}, \mathrm{G}$ ), implies tight regulation of CCDC11 levels in the cell, presumably through a missing motif on the $\mathrm{C}$-terminal part of the protein. This regulation suggests that high levels of CCDC11 might interfere with cilia function, which in turn may lead to improper later- 
ality determination. To address this hypothesis, we overexpressed $x$ Ccdc11 by dorsal mRNA injection at the 2- to 4-cell stage. To verify the injection site, green fluorescent protein (GFP)-encoding mRNA was co-injected. As a negative control, only GFP mRNA was injected and $x$ Foxj1-injected embryos served as positive controls. In addition, the truncated form of $x C c d c 11$ ( $t x C c d c 11)$, which mimics the identified splice mutation in our patients as it contains only the first 330 amino acids of the protein, as well as the human full-length $C C D C 11$ ( $h C c d c 11$ ) or the truncated human form of the gene (thCcdc11) were injected. The embryos were then allowed to develop until the early neurula stage, when they were screened and sorted to validate the site of injection according to the GFP signal.

The effect on $L-R$ patterning was determined by analyzing the expression of $x$ Coco in dissected GRPs at St. 20 as described above. Quantifying the results, we found that all uninjected control embryos and $95 \%$ of the embryos injected with GFPmRNAshowed only normal $x$ Coco expression, i.e., inhibition of $x$ Coco expression on the left side (as shown in Fig. 3A), while $42 \%$ of the positive control embryos, injected with $x F o x j 1$ mRNA, presented with laterality ambiguity $-24 \%$ with uniform expression on both sides (as shown in Fig. 3B) and 18\% with reduced expression on the right side (as shown in Fig. 3C). In addition, overexpression of all four Ccdc11 forms-hCcdc11, thCcdc11, $x C c d c 11$ and $t x C c d c 11-$ resulted in laterality ambiguity in about $30 \%$ of the injected embryos $(P<0.05$, Fig. 4). Specifically, overexpression of $h C c d c 11$ resulted in $13 \%$ of the embryos with uniform expression and $18 \%$ with reduced expression on the right side, overexpression of thCcdc11 resulted in $26 \%$ of the embryos with uniform expression and $6 \%$ with reduced expression on the right side, overexpression of $x \mathrm{Ccdc11}$ resulted in $17 \%$ of the embryos with uniform expression and $13 \%$ with reduced expression on the right side, and overexpression of $t x C c d c 11$ resulted in $11 \%$ of the embryos with uniform expression and $13 \%$ with reduced expression on the right side.

To determine whether these effects of overexpression persist in later stages and throughout L-R patterning, we overexpressed $x C c d c 11$ and analyzed the embryos for $x$ Pitx 2 expression during the tailbud stage. While $79 \%$ of the control embryos presented normal unilateral expression on the left side, and only $10 \%$ had bilateral expression and $11 \%$ had no expression, only $54 \%$ of the embryos injected with $x$ Ccdc11 showed normal $x$ Pitx2 expression, $23 \%$ had no $x$ Pitx2 expression, $21 \%$ had bilateral expression and $3 \%$ had inversed expression of $x$ Pitx2. Taken together, results showed that overexpression of Ccdc11 impairs L-R patterning. In addition, overexpression of the truncated proteins $t x C c d c 11$ and thCcdc11, corresponding to the splice mutation identified in the patients, resulted in the retention of some biological activity and in interference with axial patterning. Thus, although CCDC11 is required for axial patterning, as demonstrated by the MO experiments, its expression level must be tightly regulated to maintain proper axial patterning.

\section{Discussion}

In the era of genome high-throughput sequencing analysis, identification of novel mutations in patients is becoming routine for the geneticist. However, proving the pathogenicity of an identified mutation and understanding the etiology of the disease remains a bottleneck in the diagnostic procedure for rare diseases. For consanguineous families, the use of homozygosity mapping has proven to be a powerful tool to reduce the list of candidate pathogenic mutations (Edvardson et al., 2012 a,b). The initial identification of the mutation in CCDC11 was based on homozygosity mapping, which was followed by screening the candidate genes using gene-ontology tools and conventional Sanger sequencing (Perles et al., 2012). This screen resulted in the identification of a homozygous splice-site mutation affecting the first nucleotide of intron 6 , and resulting in the skipping of exon 6 and generation of a transcript encoding a truncated $\sim 35-k D a$ protein lacking 150 amino acids at its $\mathrm{C}$ terminus. Recently, three additional mutations in CCDC11 in unrelated patients have been described (Narasimhan et al., 2015; Noël et al., 2016). Our two sibling patients presented with different phenotypes. Similarly, the other patients presented other manifestations of laterality defects, such as dextrocardia (Noël et al., 2016), HS or SIT. Among them, one presented with mild symptoms of PCD (Narasimhan et al., 2015). It is possible that the different mutations result in different CCDC11 protein variants with different residual activities. The time window of activity, during the early stages of embryogenesis, is likely to contribute to this variability. Moreover, the lack of PCD symptoms suggests that during later stages of development and adulthood, there is a compensatory or redundancy mechanism in motile cilia, which is inactive in the node during neurulation. Finally, the identification of other patients presenting with lateralization defects emphasizes a highly important role for CCDC11 in regulating organ lateralization.

To investigate the effect of the mutation in CCDC11 on ciliogenesis, we measured the length of the cilia in control and patient skin fibroblast cells, and found that in the latter they are significantly longer, albeit less abundant. This difference has been shown to be of clinical relevance in ciliopathies (Walczak-Sztulpa et al., 2010). However, despite these differences, no clinical manifestation was observed in the ISIT patient. Notably, the cultured patient-derived skin fibroblast cells grown in vitro may have little clinical relevance to PCD. Moreover, to identify the subcellular localization of the WT and mutated truncated proteins, we immunostained the cells and found that both forms are localized to the actin cytoskeleton as well as to the centriole, the latter in agreement with a recent study (Silva et al., 2016). On the other hand, we were unable to demonstrate localization of CCDC11 to the cilia proper as previously published (Narasimhan et al., 2015). Moreover, the fact that the truncated protein also localized to the centriole and the actin cytoskeleton suggests that the motifs required for this localization reside within the $\mathrm{N}$-terminal region of the protein. Identifying this motif should be a subject of future studies.

Accumulation of the truncated protein in the ISIT patient cells was previously demonstrated by western blot (Perles et al., 2012), and was further confirmed in this study using IHC staining. This finding suggests that the expression level of the WT CCDC11 is tightly controlled and that the motif that is required for maintaining the proper level resides within the C-terminal domain of CCDC11, which is absent in the truncated form. Interestingly, treatment of control skin fibroblast cells with MG-132, a highly potent proteasome inhibitor, resulted in increased levels of CCDC11 (not shown), suggesting that the protein undergoes ubiquitin-mediated degradation (Hershko and Ciechanover, 1998). However, given that the ISIT patient was otherwise healthy, as were the heterozygous parents and five siblings, overexpression of the truncated form has no clinical significance following the L-R-determination process in the embryo. Thus, the experimental goals were set to study the 
effects of overexpression of both the WT and truncated CCDC11, as well as downregulation of the gene in frog embryos at the relevant stages of the lateralization process.

To better evaluate the roles of CCDC11 in L-R axial patterning, we used the $X$. laevis model to study the expression pattern of the gene, the mechanism regulating its expression, and the effects of knocking down or overexpressing both the WT and mutated proteins. At the mRNA level, we found that $x C c d c 11$ begins to increase in early gastrula (St.10.5) and later on, in-situ staining revealed the gene's expression in ciliated cells in the GRP (St. 17) and in ciliated ectodermal cells during the tailbud stage. Accordingly, the same expression pattern was observed at the protein level as demonstrated by IHC staining, thus confirming the specificity of the antibody to the frog ortholog. The increase in $x$ Ccdc11 coincided with the expression of $x$ Fox J1, the master regulator of the ciliogenesis program (Yu et al., 2008). We showed directly that similar to the other known downstream targets, Tekt2 and Dnah9, xCcdc11 level is also regulated by FoxJ1. Supporting this conclusion, in a microarray screen, Ccdc11 was revealed as a target gene of FoxJ1 in fish as well (Choksi et al., 2014), thereby highlighting the evolutionary conservation of the process. Taken together, our data confirm that the spatiotemporal expression pattern of $x$ Ccdc11, at both the mRNA and protein levels, in the GRP coincides with the lateralization process.

During embryogenesis, the first morphological sign of L-R patterning is the formation of the asymmetric heart tube. However, at the molecular level, the process of lateralization occurs during earlier developmental stages, starting at neurulation. The earliest known molecular manifestation of laterality is downregulation of the Nodal (Xnr1) inhibitor $x$ Coco on the left side of the GRP, which permits Xnr1 activity on this side. This cilium-driven, flow-dependent process allowed us to evaluate the effect of knocking down $x$ Ccdc11 levels during the earliest stages of laterality specification (Schweickert et al., 2010). Our results showed that $49 \%$ of the xCcdc11-MO-injected embryos showed deviation from the expected normal expression pattern of $x \mathrm{CocO}$, significantly higher than the control-MO-injected and uninjected control groups. The distribution between the phenotypes, i.e., situs ambiguity and inverted situs, was about $50 \%$, which may reflect differences in the degree of xCcdc11 knockdown or technical procedural variation. The study of Narasimhan et al., (2015) described zebrafish embryos injected with either start or splice xCcdc11-MOs. These embryos showed a curved body axis phenotype, which may be indicative of motile cilia dysfunction. Curiously, no data were provided on the efficacy of the MO-mediated Ccdc11 knockdown at the protein level, despite the availability of an antibody. Interestingly, in the study of Silva et al., (2016) which used transcription activator-like effector nucleases (TALENs) to target $C c d c 11$ expression, dissimilar phenotypes, such as pericardial edema and hydrocephalus, were described. These discrepancies between the different phenotypes probably reflect technical differences between the studies rather than biological effects. Moreover, they do not model the phenotype presented in human patients. Much clearer results for the role of Ccdc11 in zebrafish were presented by Noël et al., (2016) who used CRISPR-Cas genome-editing technology to create a Ccdc11 mutant and showed that in contrast to the $x C c d c 11$ morphants, mutant fish have disrupted laterality without any additional morphological phenotypes. This represents a model that is more similar to human patients and highlights the advantages of using the CRISPR system in fish.
Use of $x$ Coco expression as a marker for early axial patterning in the GRP allowed us to evaluate the effect of $x$ Ccdc11 knockdown on this process and to demonstrate that $x C c d c 11$ is required for symmetry breakage during neurulation. $x$ Pitx2, which is normally expressed on the left lateral plate mesoderm, is also a useful laterality indicator during tailbud stages (Campione et al., 1999; Ryan et al., 1998). The use of $x$ Pitx 2 allows discriminating between normal situs when it is expressed on the left side, situs inversus when it is expressed on the right side and situs ambiguous when it is expressed on both sides or is absent altogether. It is worth noting that since in the absence of an instructing signal, lateralization is randomized (Kawakami et al., 2005; Nonaka et al., 1998; Supp et al., 1997), some of the embryos that present normal left expression of $x P i t x 2$ may actually be embryos that had no laterality-instructing signals during neurulation and randomly became situs solitus. It is difficult to determine the size of this group as they are indistinguishable from the normal embryos. Thus, the proportion of affected embryos is probably greater than meets the eye.

The first human mutation described in CCDC11 resulted in the formation of a truncated protein which accumulated in patient cells. The two heterozygous parents and five siblings were the only known carriers of this mutation and were all asymptomatic (Perles et al., 2012). Skin biopsies were not available from these carriers, precluding us from determining whether this accumulation also occurs in their cells. However, it suggests that the truncated form has no pathogenic effect, while it is still possible that this form retains some biological activity and the levels of WT CCDC11 are tightly controlled. The results from the overexpression experiments support this notion: overexpression of both human and Xenopus orthologs, in their full-length and truncated forms, significantly affected the lateralization process, as demonstrated using the positive control of FoxJ1 overexpression. This also shows that the human and Xenopus CCDC11 orthologs have a conserved evolutionary role.

In summary, this study provides additional evidence for the role of CCDC11 in L-R axial patterning. We show that CCDC11 is localized to the centriole and actin cytoskeleton and that the motif required for that localization probably resides within the $\mathrm{N}$-terminal part of the protein. Moreover, in patient cells, ciliogenesis is altered. Using frog embryos, we show that the spatiotemporal expression pattern of $x \mathrm{Ccdc11}$ coincides with lateralization and gene-misexpression results in abnormal $L-R$ patterning. These findings highlight the importance of CCDC11 in early stages of embryogenesis.

\section{Materials and Methods}

\section{Immunohistochemical staining}

Skin fibroblast cells were fixed in $4 \%$ paraformaldehyde in PBS, permeabilized and blocked using $0.25 \%$ Triton X-100 and $5 \%$ serum in PBS for $30 \mathrm{~min}$, and then incubated with ab84324 (Abcam) at $1 \mu \mathrm{g} / \mathrm{ml}$ for $3 \mathrm{~h}$ at room temperature. The secondary antibody used was Cy2-conjugated donkey anti-rabbit Ig $\mathrm{G}(\mathrm{H}+\mathrm{L})$ at a 1:500 dilution (Jackson ImmunoResearch). Actin cytoskeleton was stained with rhodamine phalloidin according to the manufacturer's instructions (Molecular Probes). DAPI was used for nuclear staining. Pictures were taken using an Olympus BX62 fluorescent microscope equipped with a DP72 camera and controlled by cellSens software (Olympus), which was also used for analysis and measurements.

\section{Frogs and embryo manipulation}

Xenopus laevisfrogs were purchased from Xenopus I or NASCO (Dexter, $\mathrm{MI}$ and Fort Atkinson, WI, respectively). Embryos were obtained by in-vitro fertilization, incubated in $0.1 \% \mathrm{MBSH}$, and staged according to Nieuwkoop 
and Faber (1975).

Embryos were injected at the 2- to 4-cell stage with in-vitro transcribed cap-RNA, expression plasmid or antisense MO. Expression plasmid pCS2 for Ccdc11, Foxj1 and GFP was linearized with Notl and capped RNAs were in-vitro transcribed using SP6 polymerase. Cap analog $\left(\mathrm{m} 7 \mathrm{G}\left(5^{\prime}\right) \mathrm{ppp}\left(5^{\prime}\right) \mathrm{G}\right.$, Amersham) was added to the reaction mixture at a cap:GTP ratio of 5:1. For lineage tracing, GFP mRNA was injected and embryos were sorted at the neurula stages.

Antisense MOs were obtained from Gene Tools LLC:

xCcdc11-MO: 5' GACGGCTTCTTTGACTATACAGCAT 3',

control-MO: 5' CCTCTTACCTCAGTTACAATTTATA 3'.

\section{qPCR analysis}

Total RNA was extracted from embryos with PerfectPure RNA Tissue Kit with DNase (5 Prime). cDNA was synthesized using iScript cDNA Synthesis Kit (Bio-Rad). The real-time PCRs were performed using CFX384 Real Time SYStem (Bio-Rad) and Light Cycler 480 SYBR Green I Master (Roche). Samples were analyzed in triplicate and GAPDH was used as the reference housekeeping gene. Each experiment was repeated independently at least three times. The following primers were used: Ccdc11: 5'GAGGGAGGTGTATCGGACAA 3' and 5' CCTCTCCAGTAAGGTGCTGA 3'; Tekt2: 5' GCAGCGCCTTTCTTCTCATC 3' and 5' GGTGCTGTTGTCTTGCTCAT 3'; Dnah9: 5' AGAATCCAGCCATCCGTGAA 3' and 5' TCAGAAGGTCAGCGAGAGTT 3'; GAPDH: 5'GCTCCTCTCGCAAAGGTCAT 3' and 5'GGGCCATCCACTGTCTTCTG 3'.

\section{$x$ Ccdc11 and truncated-xCcdc11 cloning/plasmid cloning}

$x C c d c 11$ was cloned by PCR amplification from Xenopus cDNA. The PCR product was introduced into pCS2 vector through EcoRI and Xhol sites. Truncated $x C c d c 11$ ( $t x C c d c 11$ ) was cloned by PCR amplification with $5{ }^{\prime} \mathrm{PO}_{4}$-primers from the full-length plasmid, followed by T4 ligation and Dpnl digestion prior to $\mathrm{DH} 5 \alpha$ transformation. The primers used for the ligation were as follows:

forward: 5' PO4-TAGCAAGGGCGAaTCAAGGC 3',

reverse: 5' PO4-CTTGCGCTGGCGTTTCTCCTC 3'.

\section{Whole-mount in-situ hybridization and immunohistochemistry}

Whole-mount in-situ hybridization was performed as previously described (Epstein et al., 1997). Probes were prepared by in-vitro transcription using Digoxigenin Labeling Mix (Roche). Lineage tracing and double-staining were obtained by adding FITC to the one-side-injected mRNA, detecting it with anti-fluorescein antibody and staining with 5-bromo-4-chloro-3-indolyl phosphate p-toluidine salt (BCIP, Roche) and BM purple (Roche) to stain the second probe. For the $x C c d c 11$ probe, the plasmid was linearized with HindIII and transcribed with T7 polymerase. xPitx2 (Campione et al., 1999) and $x$ Coco (Schweickert et al., 2010) probes were transcribed as previously described.

For whole-mount IHC, embryos were fixed in MEMFA for $1 \mathrm{~h}$ at RT and processed according to a standard protocol (Sive et al., 2000). Rabbit polyclonal anti-CCDC11 antibody was diluted to a final concentration of $2 \mu \mathrm{g} / \mathrm{ml}$ (ab84324, Abcam), horseradish peroxidase anti-rabbit antibodies were used as secondary antibodies and staining was performed by DAB detection.

\section{Western blot analysis}

Total protein was extracted from embryos using lysis buffer $(150 \mathrm{mM}$ $\mathrm{NaCl}, 50 \mathrm{mM}$ Tris pH 7.6, 1.0\% Triton X-100, 6 mM EDTA) supplemented with protease inhibitor cocktail (Sigma, Israel). Protein concentration was measured by Bradford assay (Sigma, Israel). Total protein extract $(20 \mu \mathrm{g})$ was subjected to $10 \%$ SDS-PAGE and transferred to a nitrocellulose membrane. Rabbit polyclonal anti-CCDC11 antibody, diluted to a final concentration of $1 \mu \mathrm{g} / \mathrm{ml}$ (ab84324, Abcam), was used for immunoblotting.

\section{Acknowledgements}

We wish to express our heartfelt gratitude to Martin Blum and his laboratory members for helpful discussions and support, and for kindly providing the Foxj1 expression plasmid. This work was supported by grant 1255/13 from the Israel Science Foundation, founded by the Israeli Academy of Sciences (to Y.C.).

\section{References}

AFZELIUS BA (1976). A human syndrome caused by immotile cilia. Science 193: 317-319.

BARTRAM U, WIRBELAUER J, SPEER CP (2005). Heterotaxy syndrome -- asplenia and polysplenia as indicators of visceral malposition and complex congenital heart disease. Biol Neonate 88: 278-290.

BLUM M, ANDRE P, MUDERS K, SCHWEICKERT A, FISCHER A, BITZER E, BOGUSCH S, BEYER T, VAN STRAATEN HWM, VIEBAHN C (2007). Ciliation and gene expression distinguish between node and posterior notochord in the mammalian embryo. Differentiation 75: 133-146.

BLUM M, BEYER T, WEBER T, VICK P, ANDRE P, BITZER E, SCHWEICKERT A (2009). Xenopus, an ideal model system to study vertebrate left-right asymmetry. Dev Dyn 238: 1215-1225.

BLUM M, FEISTELK, THUMBERGERT, SCHWEICKERTA(2014). The evolution and conservation of left-right patterning mechanisms. Development 141: 1603-1613.

BRUECKNER M (2007). Heterotaxia, congenital heart disease, and primary ciliary dyskinesia. Circulation 115: 2793-2795.

CAMPIONE M, STEINBEISSER H, SCHWEICKERT A, DEISSLER K, VAN BEBBER F, LOWE LA, NOWOTSCHIN S, VIEBAHN C, HAFFTER P, KUEHN MR, BLUM $M$ (1999). The homeobox gene Pitx2: mediator of asymmetric left-right signaling in vertebrate heart and gut looping. Development 126: 1225-1234.

CHOKSI SP, BABU D, LAU D, YU X, ROY S, AFZELIUS BA, AUSTIN-TSE C, HALBRITTER J, ZARIWALA MA, GILBERTI RM, et al., (2014). Systematic discovery of novel ciliary genes through functional genomics in the zebrafish. Development 141: 3410-3419.

EDVARDSON S, CINNAMON Y, JALAS C, SHAAG A, MAAYAN C, AXELROD FB, ELPELEG O (2012). Hereditary sensory autonomic neuropathy caused by a mutation in dystonin. Ann Neurol 71: 569-572.

EDVARDSON S, CINNAMONY, TA-SHMAA, SHAAG A, YIM Y-I, ZENVIRT S, JALAS C, LESAGE S, BRICEA, TARABOULOSA, KAESTNER KH, GREENE LE, ELPELEG O (2012). A deleterious mutation in DNAJC6 encoding the neuronal-specific clathrin-uncoating co-chaperone auxilin, is associated with juvenile parkinsonism. PLoS One 7: e36458. http://www.ncbi.nlm.nih.gov/pubmed/22563501.

ENDICOTT SJ, BASU B, KHOKHA M, BRUECKNER M, BAHE S, STIERHOF Y-D, WILKINSON CJ, LEISS F, NIGG EA, BAYLISS R, et al., (2015). The NIMA-like kinase Nek2 is a key switch balancing cilia biogenesis and resorption in the development of left-right asymmetry. Development 142: 4068-4079.

EPSTEIN M, PILLEMER G, YELIN R, YISRAELI JK, FAINSOD A (1997). Patterning of the embryo along the anterior-posterior axis: the role of the caudal genes. Development 124: 3805-3814.

ESSNER JJ, AMACK JD, NYHOLM MK, HARRIS EB, YOST HJ (2005). Kupffer's vesicle is a ciliated organ of asymmetry in the zebrafish embryo that initiates left-right development of the brain, heart and gut. Development 132: 1247-1260.

HERSHKO A, CIECHANOVER A (1998). The ubiquitin system. Annu Rev Biochem 67: 425-479.

HIROKAWA N, TANAKA Y, OKADA Y, TAKEDA S (2006). Nodal flow and the generation of left-right asymmetry. Cell 125: 33-45.

KARTAGENER M, STUCKIP (1962). Bronchiectasis with situs inversus. Arch Pediatr 79: 193-207.

KAWAKAMI Y, RAYA Á, RAYA RM, RODRÍGUEZ-ESTEBAN C, BELMONTE JCI (2005). Retinoic acid signalling links left-right asymmetric patterning and bilaterally symmetric somitogenesis in the zebrafish embryo. Nature 435: 165-171.

KENNEDY MP, OMRAN H, LEIGH MW, DELL S, MORGAN L, MOLINA PL, ROBINSON B V, MINNIX SL, OLBRICH H, SEVERIN T, AHRENS P, LANGE L, MORILLAS HN, NOONE PG, ZARIWALA MA, KNOWLES MR (2007). Congenital heart disease and other heterotaxic defects in a large cohort of patients with primary ciliary dyskinesia. Circulation 115: 2814-2821.

LEIGH MW, PITTMAN JE, CARSON JL, FERKOL TW, DELL SD, DAVIS SD, KNOWLES MR, ZARIWALA MA (2009). Clinical and genetic aspects of primary ciliary dyskinesia/Kartagener syndrome. Genet Med 11: 473-487. 
LEVIN M (2005). Left-right asymmetry in embryonic development: a comprehensive review. Mech Dev 122: 3-25.

NARASIMHAN V, HJEIJ R, VIJS, LOGESNT, WALLMEIERJ, KOERNER-RETTBERG C, WERNER C, THAMILSELVAM SK, BOEY A, CHOKSI SP, PENNEKAMP P, ROY S, OMRAN H (2015). Mutations in CCDC11, which encodes a coiled-coil containing ciliary protein, causes situs inversus due to dysmotility of monocilia in the left-right organizer. Hum Mutat 36: 307-318.

NIEUWKOOP PD, FABER J (1975). Normal Table of Xenopus laevis (Daudin)., II. North-Holland Publishing Company, Amsterdam.

NOËL ES, MOMENAH TS, AL-DAGRIRI K, AL-SUWAID A, AL-SHAHRANI S, JIANG H, WILLEKERS S, OOSTVEEN YY, CHOCRON S, POSTMAA V, BHUIYAN ZA, BAKKERS J (2016). A Zebrafish Loss-of-Function Model for Human CFAP53 Mutations Reveals Its Specific Role in Laterality Organ Function. Hum Mutat 37: 194-200.

NONAKA S, TANAKA Y, OKADA Y, TAKEDA S, HARADA A, KANAI Y, KIDO M, HIROKAWA N (1998). Randomization of left-right asymmetry due to loss of nodal cilia generating leftward flow of extraembryonic fluid in mice lacking KIF3B motor protein. Cell 95: 829-837.

PERLES Z, CINNAMON Y, TA-SHMA A, SHAAG A, EINBINDER T, REIN AJJT, ELPELEG O (2012). A human laterality disorder associated with recessive CCDC11 mutation. J Med Genet 49: 386-390.

PERLOFF JK (2011). The Cardiac Malpositions. Am J Cardiol 108: 1352-1361.

POHL BS, KNÖCHEL W (2004). Isolation and developmental expression of Xenopus FoxJ1 and FoxK1. Dev Genes Evol 214: 200-205.

RAYAA, IZPISÚA BELMONTE JC (2006). Left-right asymmetry in the vertebrate embryo: from early information to higher-level integration. Nat Rev Genet 7:283-293.

RYANAK, BLUMBERG B, RODRIGUEZ-ESTEBAN C, YONEI-TAMURAS, TAMURA K, TSUKUI T, DE LA PEÑA J, SABBAGH W, GREENWALD J, CHOE S, NORRIS DP, ROBERTSON EJ, EVANS RM, ROSENFELD MG, BELMONTE JCI (1998). Pitx2 determines left-right asymmetry of internal organs in vertebrates. Nature 394: 545-551.

SCHWEICKERT A, VICK P, GETWAN M, WEBER T, SCHNEIDER I, EBERHARDT M, BEYER T, PACHUR A, BLUM M (2010). The nodal inhibitor coco is a critical target of leftward flow in Xenopus. Curr Biol 20: 738-743.

SCHWEICKERT A, WEBER T, BEYER T, VICK P, BOGUSCH S, FEISTEL K, BLUM $M$ (2007). Cilia-driven leftward flow determines laterality in Xenopus. Curr Biol 17: $60-66$.

SHOOK DR, MAJER C, KELLER R (2004). Pattern and morphogenesis of presumptive superficial mesoderm in two closely related species, Xenopus laevis and Xenopus tropicalis. Dev Biol 270: 163-185.

SILVA E, BETLEJA E, JOHN E, SPEAR P, MORESCO JJ, ZHANG S, YATES JR, MITCHELL BJ, MAHJOUB MR (2016). Ccdc11 is a novel centriolar satellite protein essential for ciliogenesis and establishment of left-right asymmetry. $\mathrm{Mol}$ Biol Cell 27: 48-63.

SIVE H, GRAINGER R, HARLAND R (2000). Early Development of Xenopus laevis: A Laboratory Manual. Cold Spring Harbor Laboratory Press, Cold Spring Harbor, NY.

STUBBS JL, OISHI I, IZPISÚA BELMONTE JC, KINTNER C (2008). The forkhead protein Foxj1 specifies node-like cilia in Xenopus and zebrafish embryos. Nat Genet 40: 1454-1460.

SUPPDM, WITTEDP, POTTER SS, BRUECKNERM (1997). Mutation of an axonemal dynein affects left-right asymmetry in inversus viscerum mice. Nature389:963-966.

VICK P, SCHWEICKERT A, WEBER T, EBERHARDT M, MENCL S, SHCHERBAKOV D, BEYER T, BLUM M (2009). Flow on the right side of the gastrocoel roof plate is dispensable for symmetry breakage in the frog Xenopus laevis. Dev Biol 331: 281-291.

WALCZAK-SZTULPA J, EGGENSCHWILER J, OSBORN D, BROWN DA, EMMA F, KLINGENBERG C, HENNEKAM RC, TORRE G, GARSHASBI M, TZSCHACHA SZCZEPANSKAM, KRAWCZYNSKIM, ZACHWIEJAJ, ZWOLINSKAD, BEALES PL, ROPERS H-H, LATOS-BIELENSKA A, KUSS AW (2010). Cranioectodermal Dysplasia, Sensenbrenner syndrome, is a ciliopathy caused by mutations in the IFT122 gene. Am J Hum Genet 86: 949-956.

WESSELY O, OBARA T (2008). Fish and frogs: models for vertebrate cilia signaling Front Biosci 13: 1866-1880.

YU X, NG CP, HABACHER H, ROY S (2008). Foxj1 transcription factors are master regulators of the motile ciliogenic program. Nat Genet 40: 1445-1453. 


\section{Further Related Reading, published previously in the Int. J. Dev. Biol.}

Left-right patterning in Xenopus conjoined twin embryos requires serotonin signaling and gap junctions Laura N. Vandenberg, Douglas J. Blackiston, Adam C. Rea, Timothy M. Dore, and Michael Levin Int. J. Dev. Biol. (2014) 58: 799-809

http://www.intjdevbiol.com/web/paper/140215ml

Spiral cleavages determine the left-right body plan by regulating Nodal pathway in monomorphic gastropods, Physa acuta Masanori Abe, Hiromi Takahashi and Reiko Kuroda

Int. J. Dev. Biol. (2014) 58: 513-520

http://www.intjdevbiol.com/web/paper/140087rk

Generating asymmetries in the early vertebrate embryo: the role of the Cerberus-like family

José António Belo, Ana C. Silva, Ana-Cristina Borges, Mário Filipe, Margaret Bento, Lisa Gonçalves, Marta Vitorino, Ana-Marisa Salgueiro, Vera Texeira, Ana T. Tavares and Sara Marques

Int. J. Dev. Biol. (2009) 53: 1399-1407

http://www.intjdevbiol.com/web/paper/072297jb

Xenopus nodal related-1 is indispensable only for left-right axis determination

Ryuji Toyoizumi, Tsuyoshi Ogasawara, Shigeo Takeuchi and Kazue Mogi

Int. J. Dev. Biol. (2005) 49: 923-938

http://www.intjdevbiol.com/web/paper/052008rt

Xenopus neurula left-right asymmetry is respeficied by microinjecting TGF-beta5 protein

Kazue Mogi, Madoka Goto, Eri Ohno, Yoshitaka Azumi, Shigeo Takeuchi and Ryuji Toyoizumi

Int. J. Dev. Biol. (2003) 47: 15-29

http://www.intjdevbiol.com/web/paper/12653248

Role of the anterior visceral endoderm in restricting posterior signals in the mouse embryo.

A Perea-Gomez, M Rhinn and S L Ang

Int. J. Dev. Biol. (2001) 45: 311-320

http://www.intjdevbiol.com/web/paper/11291861

Nodal signaling and the zebrafish organizer.

A F Schier and W S Talbot

Int. J. Dev. Biol. (2001) 45: 289-297

http://www.intjdevbiol.com/web/paper/11291859

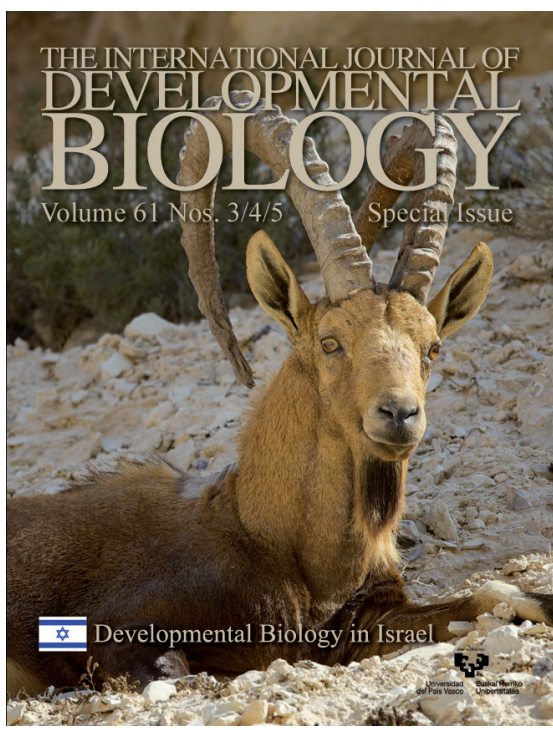

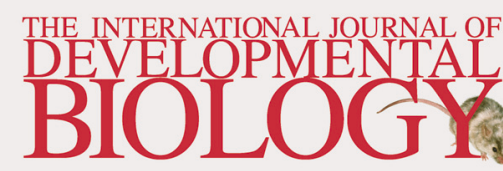

Volume 52 Nos. $2 / 3$

2008

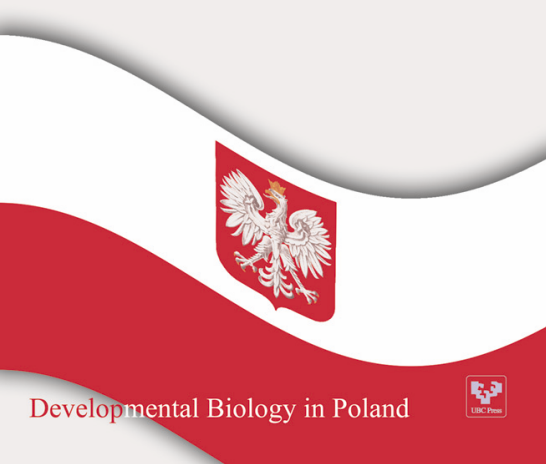

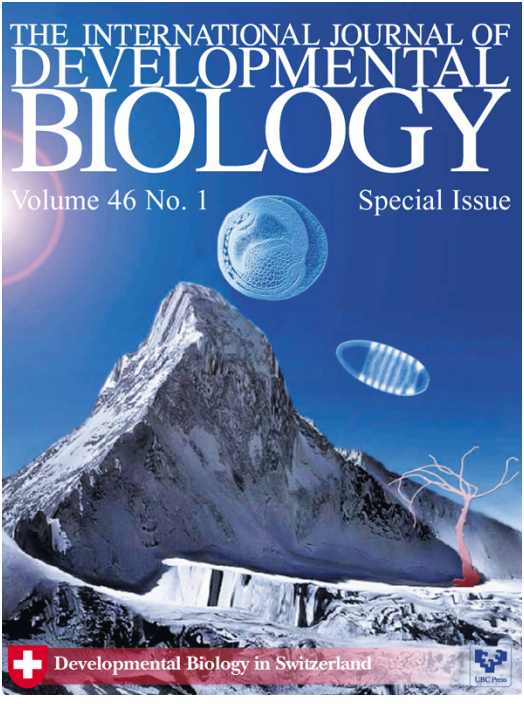

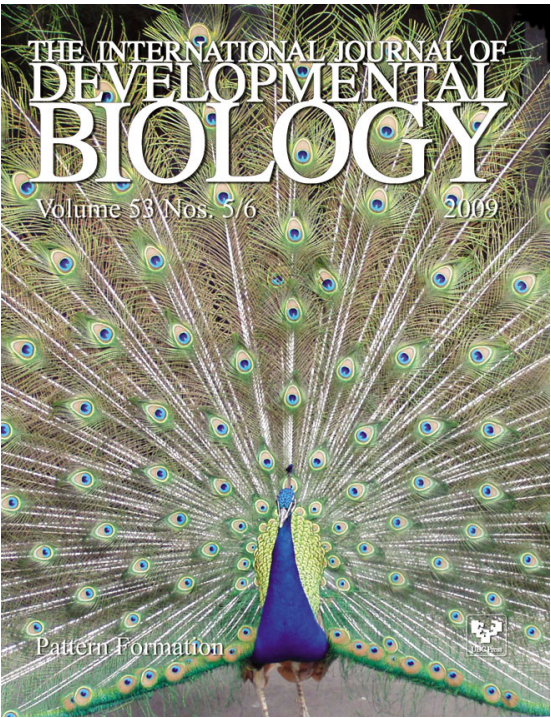

